Banca IMI, PDG internal report

(Reduced version in Finance \& Stochastics 4, pp. 147-159, February 2000)

\title{
Discrete Time vs Continuous Time Stock-price Dynamics and implications for Option Pricing *
}

\author{
Damiano Brigo \\ Fabio Mercurio \\ Product and Business Development Group \\ Banca IMI, San Paolo IMI Group \\ Corso Matteotti 6 \\ 20121 Milano, Italy \\ Fax: 390276019324 \\ E-mail: brigo@bancaimi.it_fmercurio@bancaimi.it
}

\begin{abstract}
In the present paper we construct stock price processes with the same marginal lognormal law as that of a geometric Brownian motion and also with the same transition density (and returns' distributions) between any two instants in a given discrete-time grid.

We then illustrate how option prices based on such processes differ from Black and Scholes', in that option prices can be either arbitrarily close to the option intrinsic value or arbitrarily close to the underlying stock price.

We also explain that this is due to the particular way one models the stock-price process in between the grid time instants which are relevant for trading.
\end{abstract}

${ }^{*}$ We are grateful to Aleardo Adotti, our current head at the Product Development Group of IMI/San Paolo, and to Renzo Avesani, our former head at Risk Management of Cariplo, for encouraging us in the prosecution of the most speculative side of research in mathematical finance. We are grateful also to Wolfgang Runggaldier and to two anonymous referees for their remarks and suggestions. Finally, the paper has been improved both in exposition and contents thanks to private communications with Prof. Hans Nieuwenhuis from the University of Groningen 
The theoretical result concerning scalar stochastic differential equations with prescribed diffusion coefficient whose densities evolve in a prescribed exponential family, on which part of the paper is based, is presented in detail.

\section{Keywords}

Stochastic Differential Equations, Fokker-Planck Equation, Exponential Families, Stock Price Models, Black and Scholes model, Option Pricing, Trading Time Grid, $\Delta$-Markovianity, Market Incompleteness, Option replication error.

\section{Introduction}

Since the seminal papers by Black and Scholes (1973) and Merton (1973), many researchers have tackled the problem of option pricing and hedging by resorting to continuous-time mathematics to fully exploit the richness of its theoretical results. In particular, the underlying stock price dynamics has often been modelled through a geometric Brownian motion. This assumption is still quite popular, especially among practitioners, even though the empirical distribution of stock returns is often found more leptokurtic than the normal one. Indeed, the tradeoff between analytical tractability and empirical findings still makes the geometric Brownian motion a quite acceptable choice.

However, as we shall prove in this paper, the geometric Brownian motion is not the only continuous-time process that possesses a lognormal marginal distribution and normal logreturns. In fact, we shall construct a family of processes with these characteristics and then we shall also analyse the main implications of the existence of such a family as far as option pricing is concerned.

In order to achieve our goal, we consider scalar nonlinear diffusion processes whose densities evolve in given finite-dimensional exponential families, as from Chapter 7 of Brigo (1996). We begin by a stochastic differential equation (SDE) a la Black and Scholes for the stock price, whose solution is the traditional geometric Brownian motion. The probability density of such a stock price model evolves in the exponential family of log-normal densities. It is then possible to define a second SDE with a different (and arbitrarily given) diffusion coefficient such that its solution has the same marginal lognormal density as that of the Black and Scholes (1973) geometric Brownian motion.

Furthermore, one can choose the drift of this second SDE in such a way that its solution has transition densities, between two any instants of a prescribed finite subset of the time interval, that match the transition densities of the Black and Scholes process. We thus derive a family of stock price processes that behave almost equivalently to a geometric Brownian motion in that they are probabilistically identical along the finite subset of the time interval.

We then consider the problem of option pricing in a continuous-time framework. The 
interesting result we obtain is that notwithstanding the above mentioned affinity with the Black and Scholes model, the stock price processes of our family lead to completely arbitrary option prices, ranging the whole interval of prices inbetween the no-arbitrage lower and upper bounds. Such a seeming paradox can be explained in terms of the differences between the dynamics of these stock price processes at an infinitesimal level.

Our result is similar in spirit to that of Rogers and Satchell (1996). However, our approach is much more constructive in that we are able to provide, under the original measure, easy and explicit dynamics for the stock price that are theoretically consistent with whatever option price is observed in reality.

The practical implications of our result can be analysed by considering the case of a practitioner who has to price an option. Although the physical time he works in is discrete, he usually resorts to continuous-time mathematics modelling the stock price underlying the option through a Black and Scholes (1973) process. However, as the previous theoretical result implies, the geometric Brownian motion is only one of infinitely many processes that possess the discrete-time properties required by the practitioner. Therefore, as far as the stock price dynamics is concerned, the practitioner must view all these processes as equivalent to each other. Yet, when option pricing is taken into account, he must also be aware that they all imply different, and arbitrary, option prices. Since this paradoxical situation reveals a clear limit of the approximation of discrete time with continuous time, our practitioner can react as follows. He simply avoids the theory of market completeness and just chooses a process which is consistent with the option price he believes in. Such an option price can be either exogenously given by the market or endogenously produced by a model for pricing and hedging in incomplete markets. This is a further contribution of the paper. We do not derive option prices and hedging strategies assuming a specific evolution of the underlying stock price. Instead, we define a family of processes with equivalent characteristics among which one can select a single process according to his pricing and hedging purposes.

The paper is structured as follows. In Section 2, the theoretical result on the construction of some suitable diffusion processes is briefly reviewed. Section 3 deals with the application of this result to the case of the Black and Scholes (1973) model. Section 4 analyses the problem of option pricing in continuous time and considers a natural particular case. Section 5 analyses some practical implications of using any of our alternative processes in describing the asset price dynamics. Section 6 concludes the paper.

A reduced version of this paper can be found in Brigo and Mercurio (2000). 


\section{Stochastic differential equations and exponential fam- ilies}

In this section we consider the technical result the paper is based on. This result solves the following problem for scalar diffusion processes: Given a diffusion coefficient and a curve in an exponential family of densities, find a drift such that the solution of the resulting stochastic differential equation (SDE) has a density evolving in the prescribed exponential family according to the given curve. This problem has a straightforward solution. In this section, we shall present a short summary of the steps leading to the problem formulation and to its solution. This summary is based on Chapter 7 of Brigo (1996). A related result appeared in Brigo (1997), and the general result with its applications both to mathematical finance and to stochastic nonlinear filtering is also reported in Brigo (2000).

Let us consider the SDE

$$
d X_{t}=f_{t}\left(X_{t}\right) d t+\sigma_{t}\left(X_{t}\right) d W_{t}
$$

where $\left\{W_{t}, t \geq 0\right\}$ is a standard Brownian motion independent of the initial condition $X_{0}$. In order to make sure that we are dealing with an equation whose solution exists unique, we formulate the following assumption.

(A1) The initial condition $X_{0}$ is a continuous random variable with density $p_{0}(x)>0$ for all $x \in \mathbf{R}$ w.r.t. the Lebesgue measure on $\mathbf{R}$ whose moments of any order are finite. Moreover, the stochastic differential equation (1) characterized by the coefficients $f, \sigma$, and by the initial condition $X_{0}$ admits a unique strong solution.

Explicit conditions ensuring (A1) are, for example, local Lipschitz continuity and linear growth, or the Yamada-Watanabe condition (see e.g. Rogers and Williams (1987), Section $\mathrm{V}-40)$.

Once existence and uniqueness of the solution of a SDE have been established, we can analyse the distribution of its solution at all time instants. In describing the evolution of the distribution of a diffusion process, the Fokker-Planck partial differential equation is a fundamental tool. We therefore introduce the following assumption.

(A2) The unique solution $X_{t}$ of (11) admits a density $p_{t}$ that is absolutely continuous with respect to the Lebesgue measure, i.e.,

$$
\operatorname{Prob}\left\{X_{t} \in A\right\}=\int_{A} p_{t}(x) d x, \text { for all Borel sets } A,
$$

and that satisfies the Fokker-Planck equation:

$$
\frac{\partial p_{t}}{\partial t}=-\frac{\partial}{\partial x}\left(f_{t} p_{t}\right)+\frac{1}{2} \frac{\partial^{2}}{\partial x^{2}}\left(a_{t} p_{t}\right), \quad a_{t}(\cdot)=\sigma_{t}^{2}(\cdot) .
$$


Examples of assumptions on the coefficients $f, a$ and on their partial derivatives under which (A2) holds are given in the literature. See for example Stroock and Varadhan (1979) or Friedman (1975).

In order to appropriately introduce the problem we mentioned at the beginning of the section, we now present a definition of exponential family.

Definition 2.1 Let $\left\{c_{1}, \cdots, c_{m}\right\}$ be scalar functions defined on $\mathbf{R}$, such that $\left\{1, c_{1}, \cdots, c_{m}\right\}$ are linearly independent, have at most polynomial growth, are twice continuously differentiable and the convex set

$$
\Theta_{0}:=\left\{\theta=\left\{\theta^{1}, \ldots, \theta^{m}\right\}^{\prime} \in \mathbf{R}^{m}: \psi(\theta)=\log \int \exp \left[\theta^{\prime} c(x)\right] d x<\infty\right\}
$$

has non-empty interior, where $c(x)=\left\{c_{1}(x), \cdots, c_{m}(x)\right\}^{\prime}$ and " " denotes transposition. Then

$$
E M(c)=\{p(\cdot, \theta), \theta \in \Theta\}, \quad p(x, \theta):=\exp \left[\theta^{\prime} c(x)-\psi(\theta)\right],
$$

where $\Theta \subseteq \Theta_{0}$ is open, is called an exponential family of probability densities.

Our problem consists in finding a SDE whose solution $X_{t}$ has a density $p_{t}$ that follows a prescribed curve in a given exponential family. More precisely, we require the curve $t \mapsto p_{t}$, in the space of all densities, to coincide with a given curve $t \mapsto p\left(\cdot, \theta_{t}\right)$ in a given $E M(c) !$

This is formalized in the following.

Problem 2.2 Let be given an exponential family $E M(c)$, an initial density $p_{0}$ contained in $E M(c)$, and a diffusion coefficient $a_{t}(\cdot):=\sigma_{t}^{2}(\cdot), t \geq 0$. Let $\mathcal{U}\left(p_{0}, \sigma\right)$ denote the set of all drifts $f$ such that $p_{0}, f$ and $\sigma$ and its related SDE (1) satisfy assumptions (A1) and (A2). Assume $\mathcal{U}\left(p_{0}, \sigma\right)$ to be non-empty.

Then, given the curve $t \mapsto p\left(\cdot, \theta_{t}\right)$ in $E M(c)$ (where $t \mapsto \theta_{t}$ is a $C^{1}$-curve in the parameter space $\Theta)$, find a drift in $\mathcal{U}\left(p_{0}, \sigma\right)$ whose related $S D E$ has a solution with density $p_{t}=p\left(\cdot, \theta_{t}\right)$.

The solution of this problem is given by the following.

Theorem 2.3 (Solution of Problem 2.2) Assumptions and notation of Problem 2.2 in force. Consider the stochastic differential equation

$$
\begin{aligned}
d Y_{t}= & u_{t}^{\sigma}\left(Y_{t}\right) d t+\sigma_{t}\left(Y_{t}\right) d W_{t}, \quad Y_{0} \sim p_{0} \\
u_{t}^{\sigma}(x):= & \frac{1}{2} \frac{\partial a_{t}}{\partial x}(x)+\frac{1}{2} a_{t}(x) \theta_{t}^{\prime} \frac{\partial c}{\partial x}(x) \\
& -\left(\frac{d}{d t} \theta_{t}^{\prime}\right) \int_{-\infty}^{x}\left(c(\xi)-\nabla_{\theta} \psi\left(\theta_{t}\right)\right) \exp \left[\theta_{t}^{\prime}(c(\xi)-c(x))\right] d \xi
\end{aligned}
$$

\footnotetext{
${ }^{1}$ In order to contain space and notation the underlying geometric setup is not fully developed here. We just say that the problem originated from the use of differential geometry and statistics for the nonlinear filtering problem. The reader interested in geometric aspects and other details is referred to Brigo (1996), Brigo, Hanzon and Le Gland (1999), or to the tutorial in Brigo (1999).
} 
where $\nabla_{\theta} \psi\left(\theta_{t}\right)=\left\{\partial \psi / \partial \theta^{1}\left(\theta_{t}\right), \ldots, \partial \psi / \partial \theta^{m}\left(\theta_{t}\right)\right\}^{\prime}$, with the symbol " " to be read as "distributed as".

If $u^{\sigma} \in \mathcal{U}\left(p_{0}, \sigma\right)$, then the $S D E$ (3) solves Problem 2.2, in that

$$
p_{Y_{t}}(x)=\exp \left[\theta_{t}^{\prime} c(x)-\psi\left(\theta_{t}\right)\right], \quad t \geq 0
$$

The proof of the theorem is rather straightforward. It is sufficient to write the Fokker-Planck equation for the SDE (3) and, after lengthy computations, verify that indeed

$$
\frac{\partial}{\partial t} \exp \left[\theta_{t}^{\prime} c(x)-\psi\left(\theta_{t}\right)\right]=-\frac{\partial}{\partial x}\left(u_{t}^{\sigma}(x) \exp \left[\theta_{t}^{\prime} c(x)-\psi\left(\theta_{t}\right)\right]\right)+\frac{1}{2} \frac{\partial^{2}}{\partial x^{2}}\left(a_{t}(x) \exp \left[\theta_{t}^{\prime} c(x)-\psi\left(\theta_{t}\right)\right]\right)
$$

by substituting the expression for $u$ given in the theorem. A different proof can be found in Chapter 7 of Brigo (1996) or in Brigo (2000), where in deriving the expression for $u$ it was tacitly assumed, as is done here, that

$$
\lim _{x \rightarrow-\infty} u_{t}^{\sigma}(x) p_{t}(x)=0 \text { for all } t \geq 0 .
$$

In the next section, we shall consider an interesting application of this theorem to the option pricing problem. Indeed, we shall use such result more as a "guiding tool" rather than applying it immediately as it stands. In particular, assumptions (A1) and (A2) will be checked directly and not via the sufficient conditions usually considered in the literature.

\section{Alternatives to the Black and Scholes model}

Let us consider the Black and Scholes (1973) stock price model,

$$
d S_{t}=\mu S_{t} d t+\bar{\sigma} S_{t} d W_{t}, \quad S_{0}=s_{0}, \quad t \in[0, T]
$$

where $s_{0}$ is a positive deterministic initial condition, and $\mu, \bar{\sigma}$ and $T$ are positive real constants.

The probability density $p_{S_{t}}$ of $S_{t}$, at any time $t>0$, is given by

$$
\begin{aligned}
p_{S_{t}}(x) & =\exp \left\{\zeta \ln \frac{x}{s_{0}}+\rho(t) \ln ^{2} \frac{x}{s_{0}}-\psi(\zeta, \rho(t))\right\}, \quad x>0, \\
\zeta & =\frac{\mu}{\bar{\sigma}^{2}}-\frac{3}{2}, \quad \rho(t)=-\frac{1}{2 \bar{\sigma}^{2} t}, \\
\psi(\zeta, \rho(t)) & =-\frac{(\zeta+1)^{2}}{4 \rho(t)}+\frac{1}{2} \ln \left(\frac{-\pi}{\rho(t)}\right)+\ln \left(s_{0}\right) .
\end{aligned}
$$

With the notation for exponential families introduced in the previous section, one writes

$$
c_{1}(x)=\ln \frac{x}{s_{0}}, \quad c_{2}(x)=\ln ^{2} \frac{x}{s_{0}}, \quad \theta_{t}=\{\zeta, \rho(t)\}^{\prime} .
$$


One might wish to model the stock price process by considering a different volatility function $\sigma$, instead of $\bar{\sigma} S_{t}$ in (4) 2 , while preserving major properties of the original process (41). The purpose of this section is then the construction of alternative stock price dynamics that differs from (4), yet sharing similar features from a probabilistic point of view.

Let us approach this problem by applying Theorem 2.3 to find a SDE with a given diffusion coefficient $\sigma_{t}(\cdot)$ and whose marginal density is equal to the marginal density of $S$ in all time instants of the time interval $\mathcal{T}=[\epsilon, T]$, where $0<\epsilon<T$ and $\epsilon$ can be chosen arbitrarily close to 0 . We remark that the density of $S$ is not concentrated in the whole real line, but in $(0,+\infty)$. However, the procedure resulting in Theorem 2.3 can be adapted in a straightforward way to the latter case once $-\infty$ is replaced by 0 .

It easily follows from (3) that the equation sought for is

$$
\begin{aligned}
d Y_{t} & =u_{t}^{\sigma}\left(Y_{t}, s_{0}, 0\right) d t+\sigma_{t}\left(Y_{t}\right) d W_{t}, \quad Y_{\epsilon}=S_{\epsilon}, \quad \epsilon \leq t \leq T \\
u_{t}^{\sigma}(x, y, \alpha) & :=\frac{1}{2} \frac{\partial a_{t}}{\partial x}(x)+\frac{1}{2} \frac{a_{t}(x)}{x}\left[\zeta+2 \rho(t-\alpha) \ln \frac{x}{y}\right]+\frac{x}{2(t-\alpha)}\left[\ln \frac{x}{y}-\frac{\zeta+1}{2 \rho(t-\alpha)}\right] .
\end{aligned}
$$

The definition of $Y$ is then extended to the whole interval $[0, T]$ by setting

$$
d Y_{t}=\mu Y_{t} d t+\bar{\sigma} Y_{t} d W_{t}, 0<t<\epsilon, Y_{0}=s_{0}
$$

In other terms, $Y$ is assumed to follow the same dynamics as the original process $S$ in $[0, \epsilon)$, and to start from the same initial condition.

The reason for splitting the interval $[0, T]$ into two subintervals is simply to avoid problems concerning the definition of the drift at time $t=0$. The variable $\alpha$ is here introduced to allow for subsequent generalizations.

In general, we cannot prove that (6) satisfies assumptions (A1) and (A2) for a prescribed $\sigma$, i.e. that $u^{\sigma} \in \mathcal{U}\left(p_{0}, \sigma\right)$ so that $\mathcal{U}\left(p_{0}, \sigma\right)$ is nonempty. Therefore, at this stage, (6) is simply providing us with a candidate solution of our problem for a generic $\sigma$.

In the following table we report some possible choices for $\sigma$ and the corresponding candidate $u^{\sigma}$. In particular, we can notice that for $\sigma_{t}(x)=\bar{\sigma} x, u_{t}^{\sigma}\left(x, s_{0}, 0\right)$ equals, as it must be, the original drift $\mu x$ of the Black and Scholes model (4).

Let us now assume that the particular $\sigma$ we are working with is such that $u^{\sigma} \in \mathcal{U}\left(p_{0}, \sigma\right)$.

We have seen so far that, by applying the results of the previous section, it is rather straightforward to produce processes with the same marginal distribution as that of (4). However, a further fundamental property of process (4) is that its log-returns between any two time instants are normally distributed, independently of the prices at the considered instants, i.e.,

$$
\ln \frac{S_{t+\delta}}{S_{t}} \sim \mathcal{N}\left(\left(\mu-\frac{1}{2} \bar{\sigma}^{2}\right) \delta, \bar{\sigma}^{2} \delta\right), \quad \delta>0, \quad t \in[0, T-\delta] .
$$

\footnotetext{
${ }^{2}$ We use the term "volatility" to denote the whole diffusion coefficient $\sigma_{t}(\cdot)$ rather than the standard deviation rate of the instantaneous return as usually done in practice.
} 


\section{Table 1}

Examples of volatility functions and corresponding candidate drift

\begin{tabular}{|c|c|}
\hline Volatility function $\sigma_{t}(x)$ & Drift $u_{t}^{\sigma}\left(x, s_{0}, 0\right)$ \\
\hline$\nu$ & $\frac{1}{2} \frac{\nu^{2}}{x}\left[\zeta+2 \rho(t) \ln \frac{x}{s_{0}}\right]+\frac{x}{2 t}\left[\ln \frac{x}{s_{0}}-\frac{\zeta+1}{2 \rho(t)}\right]$ \\
\hline$\nu \sqrt{x}$ & $\frac{\nu^{2}}{2}\left(\frac{\mu}{\bar{\sigma}^{2}}-\frac{1}{2}\right)-\frac{1}{2 t} \frac{\nu^{2}}{\bar{\sigma}^{2}} \ln \frac{x}{s_{0}}+\frac{x}{2}\left(\mu-\frac{\bar{\sigma}^{2}}{2}\right)+\frac{x}{2 t} \ln \frac{x}{s_{0}}$ \\
\hline$\nu x$ & $x\left[\frac{1}{4}\left(\nu^{2}-\bar{\sigma}^{2}\right)+\frac{\mu}{2}\left(\frac{\nu^{2}}{\bar{\sigma}^{2}}+1\right)\right]+\frac{x}{2 t} \ln \frac{x}{s_{0}}\left(1-\frac{\nu^{2}}{\bar{\sigma}^{2}}\right)$ \\
\hline $\bar{\sigma} x$ & $\mu x$ \\
\hline
\end{tabular}

Alternative models such as (6) do not share this property in general. In fact, identity of the marginal laws alone does not suffice to ensure (17), for which equality of second order laws or of transition densities would be sufficient instead. How can we obtain alternative models whose properties concerning log-returns are as close as possible to property (7)?

To tackle this issue, we have to find a compromise between our alternative model (6) and model (44). To this end, we consider a weaker version of (77) in that we restrict the set of dates for which the property holds true. Precisely, we modify the definition of $Y$ so that, given the time instants $\mathcal{T}^{\Delta}:=\{0, \Delta, 2 \Delta, \ldots, N \Delta\}, \Delta=T / N, \Delta>\epsilon$, property (7) is satisfied by $Y$ in $\mathcal{T}^{\Delta}$, i.e.

$$
\ln \frac{Y_{i \Delta}}{Y_{j \Delta}} \sim \mathcal{N}\left(\left(\mu-\frac{1}{2} \bar{\sigma}^{2}\right)(i-j) \Delta, \bar{\sigma}^{2}(i-j) \Delta\right), \quad i>j, \quad i=1, \ldots, N, \quad j=0, \ldots, N-1 .
$$

Limiting such key property to a finite set of times is not so dramatic. Indeed, only discrete time samples are observed in practice, so that once the time instants are fixed, our process $Y$ can not be distinguished from Black and Scholes process'. The issue of discrete versus continuous time will be further developed in Section 5.

The new definition of $Y$ is still based on Theorem 2.3. However, we use this theorem "locally" in each time interval $[(i-1) \Delta, i \Delta)$. This means that in such interval we define iteratively the drift $u^{\sigma}$ as in the theorem but

- we translate back the time-dependence of a time amount $(i-1) \Delta$ (thus locally restoring the dynamics of the original result) and

- we replace the distribution $p_{0}$ for the initial condition with the distribution of the final value of $Y$ relative to the previous interval.

We obtain:

$$
\begin{aligned}
& d Y_{t}=u_{t}^{\sigma}\left(Y_{t}, Y_{\alpha(t)}, \alpha(t)\right) d t+\sigma_{t}\left(Y_{t}\right) d W_{t}, \quad t \in[i \Delta+\epsilon,(i+1) \Delta), \\
& d Y_{t}=\mu Y_{t} d t+\bar{\sigma} Y_{t} d W_{t}, \text { for } t \in[i \Delta, i \Delta+\epsilon), \quad \alpha(t)=i \Delta \text { for } t \in[i \Delta,(i+1) \Delta),
\end{aligned}
$$

where $u_{t}^{\sigma}(x, y, \alpha)$ was defined in (마). 
It is clear by construction that the transition densities of $S$ and $Y$ satisfy $p_{Y_{(i+1) \Delta} \mid Y_{i \Delta}}(x ; y)=$ $p_{S_{(i+1) \Delta} \mid S_{i \Delta}}(x ; y)$. Then, starting from the equality of the marginal laws of $S$ and $Y$ in the first interval that holds by construction, we inductively obtain the equality of the marginal laws also in each other interval. As a consequence, the second order densities are also equal among consecutive instants $(i-1) \Delta$, is, i.e.,

$$
p_{\left[Y_{(i+1) \Delta}, Y_{i \Delta}\right]}(x, y)=p_{\left[S_{(i+1) \Delta}, S_{i \Delta}\right]}(x, y)
$$

It follows that

$$
\ln \frac{Y_{(i+1) \Delta}}{Y_{i \Delta}} \sim \mathcal{N}\left(\left(\mu-\frac{1}{2} \bar{\sigma}^{2}\right) \Delta, \bar{\sigma}^{2} \Delta\right), \quad i=0, \ldots, N-1
$$

At this point we remark that the process $Y$ in (9) is not a Markov process in $[0, T]$. However, it is Markov in all time instants of $\mathcal{T}^{\Delta}$. Formally,

$$
p_{Y_{m \Delta} \mid Y_{(m-1) \Delta}, Y_{(m-2) \Delta}, \ldots, Y_{0}}=p_{Y_{m \Delta} \mid Y_{(m-1) \Delta}} .
$$

This property follows from the fact that in $[(m-1) \Delta, m \Delta)$ the dynamics of the SDE defining $Y$ does not depend on $Y_{(m-2) \Delta}, \ldots, Y_{0}$, and that when such equation is considered for $t \in$ $[(m-1) \Delta, m \Delta)$, in its drift $u^{\sigma}$ the local initial condition for the entry $Y$ is set to $Y_{(m-1) \Delta}$.

From now on, we refer to markovianity in $\mathcal{T}^{\Delta}$ as to $\Delta$-Markovianity.

We finally notice that, through the $\Delta$-Markovianity, property (10) extends to any pair of instants in $\mathcal{T}^{\Delta}$, so as to yield (8) . Moreover, the inductive application of the $\Delta$-Markovianity and the identity of transition densities in the grid leads to the identity of the finite dimentional distributions of $S$ and $Y$ in the grid.

\section{Option pricing in continuous-time}

Let us now consider the process $\left\{B_{t}: t \geq 0\right\}$ whose value evolves according to

$$
d B_{t}=B_{t} r d t
$$

with $B_{0}=1$ and where $r$ is a positive real number, so that $B_{t}=\exp (r t)$. The process $B$ is assumed to describe the evolution of a money market account in a given financial market. The process $Y_{t}$ in (9) is instead assumed to model the evolution of some traded financial (risky) asset, typically a stock.

The financial market thus defined might admit arbitrage opportunities. As is well known, a sufficient condition which ensures arbitrage-free dynamics is the existence of an equivalent martingale measure with respect to the initially chosen numeraire. In this paper, we use the process $B$ as a numeraire, so that an equivalent martingale measure is a probability measure that is equivalent to the initial one, $P$, and under which the process $\left\{Y_{t} / B_{t}: t \geq 0\right\}$ is a martingale. A necessary condition for the existence of an equivalent martingale measure is the 
semimartingale property for the process $Y$. The process $Y$ is indeed a semimartingale under $P$ for sufficiently well behaved volatility functions $\sigma_{t}(\cdot)$.

We denote by $\mathcal{S}$ the set of all volatility functions $\sigma_{t}(\cdot)$ such that $u^{\sigma} \in \mathcal{U}\left(p_{0}, \sigma\right)$ and for which there exists a unique equivalent martingale measure.

The set $\mathcal{S}$ is obviously non-empty, since it contains at least the Black and Scholes volatility function $\sigma_{t}(x)=\bar{\sigma} x$. Moreover, as we will prove in the sequel, all volatilities functions of type $\nu I, \nu>0$, belong to $\mathcal{S}$, with $I$ denoting the identity map. An interesting example of volatility functions which do not belong to $\mathcal{S}$ is instead provided in the appendix.

We now assume that we have chosen $\sigma \in \mathcal{S}$ and the corresponding equivalent martingale measure $Q^{\sigma}$. Since $\left\{Y_{t} / B_{t}: t \geq 0\right\}$ is a martingale under such a measure, it easily follows that under $Q^{\sigma}$ the process $Y$ satisfies the SDE

$$
\begin{aligned}
& d Y_{t}=r Y_{t} d t+\bar{\sigma} Y_{t} d \widetilde{W}_{t}, \quad t \in[i \Delta, i \Delta+\epsilon) \\
& d Y_{t}=r Y_{t} d t+\sigma_{t}\left(Y_{t}\right) d \widetilde{W}_{t}, \quad t \in[i \Delta+\epsilon,(i+1) \Delta)
\end{aligned}
$$

where $\widetilde{W}$ is a standard Brownian motion under $Q^{\sigma}$.

Furthermore, under the assumption that i) there are no-transaction costs, ii) the borrowing and lending rates are both equal to $r$, iii) short selling is allowed with no restriction or penalty, and iv) the stock is infinitely divisible and pays no dividends, the unique no-arbitrage price for a given contingent claim $H \in L^{2}\left(Q^{\sigma}\right)$ is (see Harrison and Pliska $(1981,1983)$ )

$$
V_{t}=\frac{B_{t}}{B_{T}} E^{Q^{\sigma}}\left\{H \mid \mathcal{F}_{t}\right\}
$$

where $\left\{\mathcal{F}_{t}: t \geq 0\right\}$ denotes the filtration associated to the process $Y$.

In the special case of a European call option, the following are interesting problems to solve.

Problems 4.1 Let us assume that the given claim is a European call option, written on the stock, with maturity $T$ and strike K. Find:

$$
\begin{aligned}
& \inf _{\epsilon>0, \sigma \in \mathcal{S}} B_{T}^{-1} E^{Q^{\sigma}}\left\{\left(Y_{T}-K\right)^{+} \mid \mathcal{F}_{0}\right\}, \\
& \sup _{\epsilon>0, \sigma \in \mathcal{S}} B_{T}^{-1} E^{Q^{\sigma}}\left\{\left(Y_{T}-K\right)^{+} \mid \mathcal{F}_{0}\right\} .
\end{aligned}
$$

Solving these problems is equivalent to finding the lowest and highest theoretical price of the option for which the underlying stock price has lognormal marginal distribution and normal log-returns on the grid $\mathcal{T}^{\Delta}$, with standard deviations proportional to $\bar{\sigma}$.

If we denote by $V_{*}$ the value of the infimum in (13), and by $V^{*}$ the value of the supremum in (14), the following inequalities obviously hold:

$$
\left(s_{0}-K e^{-r T}\right)^{+} \leq V_{*} \leq V_{B S}(\bar{\sigma}) \leq V^{*} \leq s_{0}
$$


where $V_{B S}(\bar{\sigma})$ denotes the option Black and Scholes price at time 0 as determined by $\bar{\sigma}$, the volatility parameter in (44). Indeed, since $\bar{\sigma} I \in \mathcal{S}$, the central inequalities hold by definition of $V_{*}$ and $V^{*}$, whereas the first and the last ones feature respectively the well known no-arbitrage lower and upper bounds for option prices.

In the next subsection we will show that the first and last inequalities in (15) are actually equalities. To prove this statement, it will be sufficient to restrict our analysis to the class of volatilities $\left\{\sigma_{t}(x)=\nu x, \nu>0\right\}$. This result is at first sight surprising. Indeed, one would naively expect that the difference between prices implied by models which are equivalent in the $\Delta$-grid is bounded by a quantity that is somehow related to $\Delta$, typically $\mathcal{O}\left(\Delta^{\lambda}\right)$ for some positive real $\lambda$. In fact, by halving the size of $\Delta$, we double the discrete-time instants where the models in our family are equivalent. Accordingly, we would expect the prices implied by these now "closer" models to range in a narrower interval. However, as we shall soon see, this is not the case.

\subsection{A fundamental case}

We begin by stating the following.

Lemma 4.2 In the fundamental case where $\sigma_{t}(x)=\nu x, \nu>0$, the process $Y^{\nu}$ given by

$$
\begin{aligned}
d Y_{t}^{\nu} & =u_{t}^{\nu I}\left(Y_{t}, Y_{\alpha(t)}, \alpha(t)\right) d t+\nu Y_{t} d W_{t}, \quad t \in[i \Delta+\epsilon,(i+1) \Delta) \\
u_{t}^{\nu I}\left(y, y_{\alpha}, \alpha\right) & =y\left[\frac{1}{4}\left(\nu^{2}-\bar{\sigma}^{2}\right)+\frac{\mu}{2}\left(\frac{\nu^{2}}{\bar{\sigma}^{2}}+1\right)\right]+\frac{y}{2(t-\alpha)}\left(1-\frac{\nu^{2}}{\bar{\sigma}^{2}}\right) \ln \frac{y}{y_{\alpha}}, \\
d Y_{t}^{\nu} & =\mu Y_{t}^{\nu} d t+\bar{\sigma} Y_{t}^{\nu} d W_{t} \text { for } t \in[i \Delta, i \Delta+\epsilon), \quad \alpha(t)=i \Delta \text { for } t \in[i \Delta,(i+1) \Delta)
\end{aligned}
$$

solves Problem 2.2 when $p_{t}$ is given by (5). Moreover, the volatility function $\sigma_{t}(x)=\nu x$ belongs to $\mathcal{S}$, for any $\nu>0$.

Proof. Since $Y^{\nu}$ has the same marginal distribution as $S$ under $P$, it follows that $Y_{t}^{\nu}>0$. Then, the process $Z_{t}=\ln Y_{t}^{\nu}$ is well defined and, by Itô's formula, the SDE for $Z_{t}$ is piecewise linear in a narrow sense (in that its diffusion coefficient is purely deterministic), and hence admits a unique strong solution which, for each $t \in[j \Delta,(j+1) \Delta)$, is explicitly given by

$$
\begin{aligned}
Z_{t}= & Z_{j \Delta}+\left(\mu-\frac{1}{2} \bar{\sigma}^{2}\right)(t-j \Delta) \\
& + \begin{cases}\bar{\sigma}\left(W_{t}-W_{j \Delta}\right) & t \in[j \Delta, j \Delta+\epsilon), \\
\left(\frac{t-j \Delta}{\epsilon}\right)^{\beta / 2}\left[\bar{\sigma}\left(W_{j \Delta+\epsilon}-W_{j \Delta}\right)+\nu \int_{j \Delta+\epsilon}^{t}\left(\frac{u-j \Delta}{\epsilon}\right)^{-\beta / 2} d W_{u}\right] & t \in[j \Delta+\epsilon,(j+1) \Delta),\end{cases}
\end{aligned}
$$

where $\beta=1-\frac{\nu^{2}}{\bar{\sigma}^{2}}$.

As a consequence, the assumptions of Problem 2.2 are satisfied so that $u^{\nu I}$ solves Problem 2.2 when $p_{t}$ is given by (5). Moreover, the Girsanov change of measure from $P$ to $Q^{\nu I}$ is well defined 
since one can show that the Novikov condition is satisfied through application of the "tower property" of conditional expectations. Hence, the measure $Q^{\nu I}$ exists unique and $\nu I$ belongs to $\mathcal{S}$ for each $\nu>0$. For the uniqueness of the measure $Q^{\nu I}$, we refer for instance to Duffie (1996).

Theorem 4.3 In the fundamental case where $\sigma_{t}(x)=\nu x, \nu>0$, the unique no-arbitrage option price at time $t$ is the Black and Scholes price

$$
U^{\epsilon}(t, \nu)=Y_{t}^{\nu} \Phi\left(d_{1}\right)-K e^{-r(T-t)} \Phi\left(d_{2}\right),
$$

where

$$
\begin{aligned}
& d_{1}=\frac{\ln \left(Y_{t}^{\nu} / K\right)+\left(r+\bar{\nu}^{\epsilon}(t)^{2} / 2\right)(T-t)}{\bar{\nu}^{\epsilon}(t) \sqrt{T-t}}, \\
& d_{2}=d_{1}(t)-\bar{\nu}^{\epsilon}(t) \sqrt{T-t}, \\
& \bar{\nu}^{\epsilon}(t)= \begin{cases}\sqrt{\frac{\frac{\epsilon}{\underline{\underline{\alpha}}\left(\bar{\sigma}^{2}-\nu^{2}\right)(T-\alpha(t))+\nu^{2}(T-\alpha(t))+\bar{\sigma}^{2}(\alpha(t)-t)}}{T-t}} & t \in[\alpha(t), \alpha(t)+\epsilon) \\
\sqrt{\frac{\frac{\epsilon}{\underline{\underline{(}}\left(\bar{\sigma}^{2}-\nu^{2}\right)(T-\alpha(t)-\Delta)+\nu^{2}(T-t)}}{T-t}} & t \in[\alpha(t)+\epsilon, \alpha(t)+\Delta)\end{cases}
\end{aligned}
$$

Proof. From the previous lemma, we infer the existence of a unique no-arbitrage option price that can be calculated through (12). Then (18) is obtained by noticing that under the equivalent martingale measure

$$
\ln \frac{Y_{T}^{\nu}}{Y_{t}^{\nu}} \sim \mathcal{N}\left(\left(r-\frac{1}{2} \bar{\nu}^{\epsilon}(t)^{2}\right)(T-t), \bar{\nu}^{\epsilon}(t)^{2}(T-t)\right), t \in[0, T],
$$

with $\bar{\nu}^{\epsilon}$ given by (19). This implies that the option price at time $t$ corresponding to $Y^{\nu}$ is the Black and Scholes price with volatility coefficient $\bar{\nu}^{\epsilon}(t)$, i.e., that (18) holds.

The key point of our result is that, for any given volatility coefficient $\nu$, we are free to adjust the drift of the SDE defining the dynamics of the stock-price process under the objective measure, in such a way that the resulting $Y^{\nu}$ has the same distributional properties of the Black and Scholes process on discrete-time dates. As opposed to this, the risk-neutral valuation for pricing options imposes the $\operatorname{drift} r Y^{\nu}$ to the $\mathrm{SDE}$ followed by $Y^{\nu}$ under the equivalent martingale measure. This causes the option price implied by the alternative model to coincide, at first order in $\epsilon$, with the Black and Scholes price with volatility parameter $\nu$. In fact, imposing the $\operatorname{drift} r Y^{\nu}$ to $Y^{\nu}$ leads to the same risk neutral process as that of Black and Scholes' (obtained by imposing the drift $r S$ to $S$ ), with the only difference that $\bar{\sigma}$ is replaced by $\nu$.

Remark 4.4 (Historical versus Implied volatility). A first interesting property that can be deduced from this theorem applies in case one believes that option prices trade independently of the underlying stock price. We have in fact been able to construct a stock price process, 
the process (16), whose marginal distribution and transition density depend on the volatility coefficient $\bar{\sigma}$, whereas the corresponding option price, in the limit for $\epsilon \rightarrow 0$, only depends on the volatility coefficient $\bar{\nu}$. As a consequence, we can provide a consistent theoretical framework which justifies the differences between historical and implied volatility that are commonly observed in real markets.

Straightforward application of the previous theorem leads to the main result of this section which is summarized in the following.

Corollary 4.5 The solutions of problems (13) and (14) are

$$
\begin{gathered}
V_{*}=\left(s_{0}-K e^{-r T}\right)^{+} \\
V^{*}=s_{0} .
\end{gathered}
$$

Moreover, for any other candidate price $\bar{V} \in\left(V_{*}, V^{*}\right)$ there exist a volatility $\nu$ and an $\epsilon>0$ such that

$$
U^{\epsilon}(0, \nu)=\bar{V}
$$

Proof. To prove (20), we simply have to take the limit of expression (18) (with $t=0$ ) for $\epsilon$ going to zero and $\nu$ either going to zero or going to infinity, since

$$
\begin{aligned}
& \lim _{v \rightarrow 0} V_{B S}(v)=\left(s_{0}-K e^{-r T}\right)^{+}, \\
& \lim _{v \rightarrow+\infty} V_{B S}(v)=s_{0},
\end{aligned}
$$

and

$$
\lim _{\epsilon \rightarrow 0} \bar{\nu}^{\epsilon}(0)=\nu
$$

Finally, we remember that, ceteris paribus, $V_{B S}(v)$ is a strictly increasing function of $v$, so that $V_{B S}(v)=\hat{V}$ has a unique solution for $V \in\left(V_{*}, V^{*}\right)$, hence $U^{\epsilon}(0, \nu)=\bar{V}$ has a solution in $(0,+\infty) \times\left(V_{*}, V^{*}\right)$.

Remark 4.6 (Taking $\epsilon \rightarrow 0$ ). It is possible to consider the limit for $\epsilon \rightarrow 0$ in the above expressions so as to present our result in a simpler and more elegant way. However, the treatment with $\epsilon$ does not involve limit considerations and permits to contain analytical effort, so that we decided to keep $\epsilon>0$. This can be also useful in numerical implementations. We just observe that, for $\epsilon \rightarrow 0$, since $\beta<1$, 17) becomes

$$
\begin{aligned}
Z_{t}= & Z_{j \Delta}+\left(\mu-\frac{1}{2} \bar{\sigma}^{2}\right)(t-j \Delta) \\
& +(t-j \Delta)^{\beta / 2} \nu \int_{j \Delta}^{t}(u-j \Delta)^{-\beta / 2} d W_{u} \quad t \in[j \Delta,(j+1) \Delta) .
\end{aligned}
$$

This process is well defined since the integral in the right-hand side exists finite a.s. even though its integrand diverges when $u \rightarrow j \Delta^{+}$. 
The above equation can be better compared to the Black and Scholes process when written in differential form:

$d Z_{t}=\left(\mu-\frac{1}{2} \bar{\sigma}^{2}\right) d t+\frac{\beta}{2}(t-j \Delta)^{\beta / 2-1} \nu \int_{j \Delta}^{t}(u-j \Delta)^{-\beta / 2} d W_{u} d t+\nu d W_{t} \quad t \in[j \Delta,(j+1) \Delta)$.

By observing this last equation we can isolate three terms in the right-hand side. The first term is the same drift as in the log-returns of the Black and Scholes process. The third term is the same as in the log-returns of the Black and Scholes process, but the volatility parameter $\bar{\sigma}$ is replaced by our $\nu$. Finally, the central term is the term which is needed to have returns equal to the returns in the Black and Scholes process even after changing the volatility from $\bar{\sigma}$ to $\nu$. Note that this term goes to zero for $\bar{\sigma}=\nu$. It is this term that makes our process non-Markov outside the trading time grid.

The interpretation of the previous theorem and corollary is as follows. If we are given a discretely observed stock price, the particular way we use to "complete" the model with any of our continuous-time processes $Y$ has a heavy impact on the associated option price. The influence is in fact so relevant that such a price can be arbitrarily close to either no-arbitrage bounds for option prices.

From an intuitive point of view, the reason why option prices can be so different is because the time step in the grid $\mathcal{T}^{\Delta}$ is never infinitesimal. In other words, continuous-time option prices would simply reveal the differences existing at an infinitesimal level among all the stock price processes $Y^{\nu}$.

\section{Option pricing and hedging in the real world}

Let us now consider a trader who needs to price an option on a given stock. His usual practice is to resort to continuous-time mathematics to fully exploit the richness of its theoretical results, and to model stock returns with a normal distribution.

Let us denote by $\delta t$ the length of the smallest time interval when an actual transaction can occur. Such $\delta t$ is the best realistic approximation of the infinitesimal time distance " $d t$ ".

The results of the previous section imply that the geometric Brownian motion (4) is just one of the infinitely many processes $Y$ that possess the properties required by the practitioner along intervals of equal length $\delta t$. However, the basic equivalence in the description of the stock price dynamics can not be extended to the corresponding option prices. Indeed, any real number in the interval $\left(V_{*}, V^{*}\right)$ can be viewed as the unique no-arbitrage option price for some process $Y$.

Which process $Y$ should then be chosen by the practitioner?

A possible answer to this question can be provided, for example, through the estimation of the option replication error in discrete time. To this end, for any $\nu>0$, let us denote by $\left(\xi^{\nu}, \eta^{\nu}\right)$ the self-financing strategy that replicates in continuos time the option payoff for the 
process $Y^{\nu}$, where $\xi_{t}^{\nu}$ and $\eta_{t}^{\nu}$ are respectively interpreted as the number of stock shares and money units held at time $t$.

Then, fixing a set of dates $\tau_{0}=0<\tau_{1}<\cdots<\tau_{n}=T$ such that $\tau_{i}=i \delta t 3$ and, denoting the observed stock price at time $\tau_{j}$ by $\bar{S}_{\tau_{j}}, j=0, \ldots, n$, the replication error when hedging according to the strategy $\left(\xi^{\nu}, \eta^{\nu}\right)$, starting from the endowment $U^{\epsilon}(0, \nu)$, is

$$
\varepsilon(\nu):=\left(\bar{S}_{T}-K\right)^{+}-U^{\epsilon}(0, \nu)-\sum_{j=0}^{n-1} \xi_{\tau_{j}}^{\nu}\left(\bar{S}_{\tau_{j+1}}-\bar{S}_{\tau_{j}}\right)-\sum_{j=0}^{n-1} \eta_{\tau_{j}}^{\nu}\left(e^{r \tau_{j+1}}-e^{r \tau_{j}}\right)
$$

At this stage, we can solve, for example, an optimization problem where $\varepsilon(\nu)$ is minimized, according to some criterion, over all $\nu>0$. Such procedure, however, is justified only to measure the performance of our continuous-time prices and strategies on the fixed set of discrete-time instants. More generally, the issue of deriving a fair $\delta$-time option price and hedging strategy should be tackled by resorting to the existing literature on incomplete markets.

Many are the criterions one can choose from for pricing and hedging in incomplete markets. We mention for instance those of Föllmer and Sondermann (1986), Föllmer and Schweizer (1991), Schweizer (1988, 1991, 1993, 1994, 1995, 1996), Schäl (1994), Bouleau and Lamberton (1989), Barron and Jensen (1990), El Karoui, Jeanblanc-Picqué and Viswanathan (1991), Davis (1994), Frasson and Runggaldier (1997), El Karoui and Quenez (1995), Frittelli (1996), Mercurio (1996), Mercurio and Vorst (1997), Bellini and Frittelli (1997), Frey (1998) and Föllmer and Leukert (1998).

However, the purpose of this section is not to favor any particular approach. We want, instead, to stress the following innovative feature in the theoretical problem of option pricing. Instead of fixing a stock price process and then deriving a fair option price and an "optimal" hedging strategy, we can in fact consider a family of processes, that are somehow equivalent in the description of the stock price evolution, among which we can select a convenient one by means of our favorite incomplete markets criterion.

A comparison between the performances of the strategy $\left(\xi^{\nu}, \eta^{\nu}\right)$ and the hedging strategy associated to any incomplete-market criterion is beyond the scope of the paper and is left to future research.

\section{Conclusions}

In the present paper we consider an option-pricing application of a result which is based on the construction of nonlinear SDE's with densities evolving in a given finite-dimensional exponential family. Precisely, we derive a family of stock price models that behave almost equivalently to that of Black and Scholes. All such models share the same distributions for the stock price

${ }^{3}$ Although fixed a priori, the set $\mathcal{T}^{\Delta}$ introduced earlier is arbitrarily chosen, so that we can set $\Delta=\delta t / k$, with $k$ any positive integer implying that $\left\{\tau_{1}, \ldots, \tau_{n}\right\} \subset \mathcal{T}^{\Delta}$. 
process and its log-returns along any previously fixed 'trading time-grid'. Therefore, all these models can be viewed as equivalent in the description of the stock price evolution.

However, the continuous-time dynamics chosen to 'complete' the model in between the instants of the trading time-grid, reflects heavily on the option price. The option price in fact can assume any value between the option intrinsic value and the underlying stock price.

As a conclusion, our result points out that no dynamics is the right one a priori, and that an incomplete-market criterion is needed to choose among all the different models. Practitioners with different criteria can still pick up a model from our family, so as to match their expectations or to minimize their exposures.

Even though our results are based on the assumption of a lognormal distribution for the stock price and a normal distribution for its log-returns, the generalization to many other distributions is possible. Notice, indeed, that the results of Section 2 can be applied to any curve of densities in a given exponential family. However, the generalization is not straightforward and heavily relies on the particular distributions which are considered.

\section{Appendix}

In this appendix we consider the case $\sigma_{t}(\cdot) \equiv \nu \neq 0$ as an interesting example of a class of volatilities which do not belong to $\mathcal{S}$. Suppose, for the sake of simplicity, that we require only the marginal law of $Y$ to coincide with the marginal law of $S$, ignoring the returns distributions. Now assume by contradiction that $\nu \in \mathcal{S}$. Then by applying (6) we obtain a Markov process $Y$

$$
d Y_{t}=\frac{1}{2} \frac{\nu^{2}}{Y_{t}}\left[\zeta+2 \rho(t) \log \frac{Y_{t}}{s_{0}}\right] d t+\frac{Y_{t}}{2 t}\left[\log \frac{Y_{t}}{s_{0}}-\frac{\zeta+1}{2 \rho(t)}\right] d t+\nu d W_{t}, \quad \epsilon \leq t \leq T,
$$

with $Y_{t}=S_{t}$ for each $t \in[0, \epsilon]$.

Let us focus on $t \geq \epsilon$. Since we now know that $Y_{t}$ and $S_{t}$ have the same distribution under the objective probability measure, $Y_{t}$ is lognormally distributed, and in particular $Y_{t}>0$ for all $t$. Since $\nu \in \mathcal{S}$, there exists an equivalent martingale measure, so that, under such a measure,

$$
d Y_{t}=r Y_{t} d t+\nu d \widetilde{W}_{t}
$$

where $\widetilde{W}$ is a standard Brownian motion under the martingale measure. Now notice that this last SDE is a linear equation, so that its solution has a density whose support is the whole real line. In other terms, such solution can be negative with positive probability at any fixed time instant, as opposed to what we have seen for $Y$ under the objective measure. However, there cannot be an equivalent measure that transforms a process whose support is the positive halfline into another one whose support is the whole real line. Therefore, we have contradicted our assumption that $\nu \in \mathcal{S}$.

Our example is similar in spirit to that of Delbaen and Schachermayer (1995) who consider a Bessel process (taking positive values at all times) which cannot be transformed into a Brownian 
motion. Some conditions for the existence of an equivalent martingale measure are given for example in Rydberg (1997).

\section{References}

[1] BARRON, E. N. and JANSEN, R. (1990). A Stochastic Control Approach to the Pricing of Options. Mathematics of Operations Research 15, 49-79.

[2] BLACK, F. and SCHOLES, M. (1973). The Pricing of Options and Corporate Liabilities. Journal of Political Economy 81, 637-654.

[3] BELlini, F. and FRITTELLI, M. (1997). Certainty Equivalent and No Arbitrage: a Reconciliation via Duality Theory. Working Paper. Brescia University. Italy.

[4] BOUleaU, N. and LAMBERTON, D. (1989). Residual Risks and Hedging Strategies in Markovian Markets. Stochastic Processes and their Applications 33, 131-150.

[5] BRIGO, D. (1996). Filtering by Projection on the Manifold of Exponential Densities. PhD Thesis, Free University of Amsterdam.

[6] BRIGO, D. (1997). On nonlinear SDEs whose densities evolve in a finite-dimensional family, in: I. Csiszár and Gy. Michaletzky (Editors), Stochastic Differential and Difference Equations, Birkhäuser, Boston, 11-19.

[7] BRIGO, D. (1999) Diffusion Processes, Manifolds of Exponential Densities, and Nonlinear Filtering, in: O.E. Barndorff-Nielsen and E. B. Vedel Jensen (Editors), Geometry in Present Day Science, World Scientific.

[8] BRIGO, D. (2000). On SDEs with marginal laws evolving in finite-dimensional exponential families. Statistics and Probability Letters 49 , pp. 127-134

[9] BRIGO, D., HANZON, F., and LE GLAND, F. (1999). Approximate nonlinear filtering by projection on exponential manifolds of densities. Bernoulli, Vol. 5, N. 3, pp. 495-534.

[10] BRIGO, D., and MERCURIO, F. (2000). Option Pricing Impact of Alternative Continuous-Time Dynamics for Discretely-Observed Stock Prices. Finance and Stochastics, Vol. 4, N. 2.

[11] DAVIS, M. H. A. (1994). A general Option Pricing Formula. Preprint. Imperial College, London.

[12] DelBAEN, F., and SCHACHERMAYER, W. (1995). Arbitrage possibilities in Bessel processes and their relations to local martingales. Probability Theory and Related Fields 102, 357-366. 
[13] DUFFIE, D. (1996). Dynamic Asset Pricing Theory, Second Edition. Academic press, New York.

[14] EL KAROUI, N., JEANBLANC-PICQUÉ, M. and R. VISWANATHAN (1991). On the Robustness of Black-Scholes equation. Laboratoire de Mathematiques et Modelisations, Ecole Nationale des Ponts and Chaussees-Ecole Normale Superieure de Cachan, Prepublication $91 / 1$.

[15] EL KAROUI, N., and QUEnEZ, M. C. (1995). Dynamic Programming and Pricing of Contingent Claims in an Incomplete Market. SIAM Journal on Control and Optimization 33, 29-66.

[16] FÖELLMER, H. and SONDERMANN, D. (1986). Hedging of Non-Redundant Contingent Claims. In W. Hildenbrand and A. Mas-Colell (eds.), Contribution to Mathematical Economics, 205-223.

[17] FÖELlMER, H. and SCHWEIZER, M. (1991). Hedging of Contingent Claims Under Incomplete Information. In Applied Stochastic Analysis (M.H.A. Davis and R.J.Elliott eds.), Stochastic Monographs vol.5, Gordon \& Breach, London/New York, 389-414.

[18] FÖELlmer, H. and LEukERT, P. (1998). Quantile Hedging. Preprint. HumboldtUniversität Berlin.

[19] FRASSON, S. and RUNGGALDIER, W.J. (1997). A Stochastic Control Model for Hedging in Incomplete Markets. Statistics and Control of Stochastic Processes, edited by Yu. M. Kabanov, B.L.Rozovskii and A.N. Shiryaev. World Scientific. Singapore.

[20] FREY, R (1998). Superreplication in Stochastic Volatility Models and Optimal Stopping. Preprint. Department of Mathematics. ETH Zürich. Switzerland.

[21] FRIEDMAN, A. (1975). Stochastic Differential Equations and Applications, I. Academic Press, New York.

[22] FRITTELLI, M. (1996). The Minimal Entropy Martingale Measure and the Valuation Problem in Incomplete Markets. Journées Internationales de Finance, AFFI, Geneva, June 1996

[23] HARRISON, J.M. , and PLISKA, S. R. (1981). Martingales and Stochastic Integrals in the Theory of Continuous Trading. Stochastic Processes and Their Applications, 11, 215-260.

[24] HARRISON, J.M. , and PLISKA, S. R. (1983). A Stochastic Calculus Model of Continuous Trading: Complete Markets. Stochastic Processes and Their Applications, 15, 313-316.

[25] MERCURIO, F. (1996). Claim pricing and hedging under market imperfections. PhD Thesis, Tinbergen Institute, Rotterdam. 
[26] MERCURIO, F. and VORST A.C.F. (1997), Option Pricing and Hedging in Discrete Time with Transaction Costs. Mathematics of Derivative Securities, edited by M.A.H. Dempster and S.R. Pliska, 190-215.

[27] MERTON, R. (1973). Theory of Rational Option Pricing. Bell Journal of Economics and Management Science 4, 141-183.

[28] ROGERS, L.C.G. and WILLIAMS, D. (1987). Diffusions, Markov Processes, and Martingales, Vol. II. Wiley \& Sons.

[29] ROGERS, L.C.G. and SATCHELL, S.E. (1996). Does the Behaviour of the Asset Tell us Anything about the Option Price Formula? A Cautionary Tale. Preprint. University of Bath.

[30] RYDBERG, T. H. (1997). A note on the existence of unique equivalent martingale measures in a Markovian setting. Finance and Stochastics 4, 251-257.

[31] SCHÄL, M. (1994). On Quadratic Cost Criteria for Option Hedging. Mathematics of Operation Research 19, 121-131.

[32] SCHWEIZER, M. (1988). Hedging of Options in a General Semimartingale Model. Diss. ETHZ No 8615.

[33] SCHWEIZER, M. (1991). Option Hedging for Semimartingales. Stochastic Processes and their Applications 37, 339-363.

[34] SCHWEIZER, M. (1993). Approximating Random Variables by Stochastic Integrals, and Applications in Financial Mathematics. Habilitationsschrift. University of Göttingen.

[35] SCHWEIZER, M. (1994). Risk-Minimizing Hedging Strategies Under Restricted Information. Mathematical Finance 4, 327-342.

[36] SCHWEIZER, M. (1995). Variance Optimal Hedging in Discrete Time. Mathematics of Operation Research 20, 1-32.

[37] SCHWEIZER, M. (1996). Approximation Pricing and the Variance Optimal Martingale Measure. Annals of Applied Probability 24, 206-236.

[38] STROOCK, D.W. and VARADHAN, S.R.S (1979). Multidimensional diffusion processes. Springer, Berlin. 